\title{
Cultural Context of Caregiving: Differences in Depression Between Puerto Rican and Non-Latina White Mothers of Adults With Mental Retardation
}

\author{
Sandra Magaña, Marsha Mailick Seltzer, and Marty Wyngaarden Krauss
}

\begin{abstract}
Differences in depression between Puerto Rican and non-Latina White mothers providing care to their adult child with mental retardation were examined. The focus of this study is on how family problems may mediate the effect of the adult's behavior problems on the mother's level of depressive symptoms and how this process differs across the two groups of mothers. As hypothesized, family problems was a stronger predictor of depressive symptoms for Puerto Rican mothers than for nonLatina White mothers. In addition, Puerto Rican mothers were in poorer physical health, which further accounted for differences in depression between the two groups.
\end{abstract}

In this paper we examine differences and similarities in depression between Puerto Rican and non-Latina White mothers who provide in-home care to an adult son or daughter with mental retardation. Our primary purpose was to examine factors that account for differences in depressive symptoms between the two groups of mothers. We are particularly interested in whether there are cultural factors that interact with caregiving challenges and whether these cultural factors add to the explanation of group differences in depression. This research was conducted in the context of a stress and coping model that incorporates family role strain as a key variable. Our aim was to determine similarities and differences in how family role strain affects maternal depression among Latina and non-Latina White mothers because the family has been shown to be particularly important to Latino mental health (Aranda \& Knight, 1997; Baca Zinn, \& Wells, 2000).

Epidemiological researchers who have examined depression using the Center for Epidemiological Studies Depression Scale (CES-D) among Puerto Rican women in the United States have found that these women have elevated levels of depressive symptoms, with average scores ranging from 14.0 to 18.5 (Cho et al., 1993; Falcon \& Tucker, 2000; Potter, Rogler, \& Moscicki, 1995) com- pared to community samples of White women whose CES-D scores ranged from 10.9 to 11.4 (Falcon \& Tucker, 2000; Gatz \& Hurwicz, 1990). However, although depression has been identified as a serious public health problem for Puerto Rican women on the United States mainland, it has not been found to be a serious problem for women on the island of Puerto Rico (Canino, Bird, Rubio-Stipec, \& Bravo, 2000; Canino et al., 1987; Shrout et al., 1992). Canino and her colleagues conducted epidemiological studies of Puerto Ricans on the island of Puerto Rico and Whites in the United States using the Diagnostic Interview Schedule and found that prevalence rates of depression in Puerto Rico were comparable to those of the general population in the United States (Canino et al., 2000; Canino et al., 1987; Shrout et al., 1992). These findings discount assumptions that Puerto Ricans may be more depressed in general or may have a cultural tendency to report more depressive symptoms and suggest, instead, that the environmental context of living in the United States or the experience of immigration may bear some explanatory value.

Factors that have been shown to be related to elevated depression among the general United States population include low income and education levels, poor health status, female gender, and 
disrupted marital status (Falcon \& Tucker, 2000; Potter et al., 1995; Radloff, 1977). However, investigators who have compared non-Latino Whites to Puerto Ricans have shown that even controlling for these factors, significant between-group differences in major depression or depressive symptoms persist (Bassuk, Perloff, \& García Coll, 1998; Falcon \& Tucker, 2000). For example, Falcon and Tucker examined depression among a representative sample of older adults in Massachusetts (where the present study was conducted) and found that the Puerto Ricans in the sample had significantly more health problems, lower educational levels, and lower income than did non-Latino Whites. However, even when these differences were controlled statistically, the Puerto Ricans continued to have significantly more depressive symptoms than did non-Latino Whites. In a different study of Puerto Rican and non-Latino Whites in Massachusetts, Bassuk et al. compared depression rates among poor single mothers with similar levels of income, education, and health. They found that the prevalence of major depression as measured by the Structured Clinical Interview for the Diagnostic and Statistical Manual of Mental Disorders was significantly higher for the Puerto Rican mothers. Bassuk et al. attributed this difference to the stress faced by Puerto Rican mothers on the United States mainland due to social and cultural isolation and the difficulties they encounter in attempting to maintain cultural values in a different society.

Research on non-Latina White mothers caring for adults with mental retardation has shown that these mothers are similar to their noncaregiving age peers in depressive symptoms (Krauss \& Seltzer, 1993). In contrast, studies of Latina mothers of children and adults with mental retardation have shown that their depressive symptom levels are elevated as compared with those of non-Latina White mothers (Blacher, Shapiro, Lopez, Diaz, \& Fusco, 1997; Magaña, 1999). High levels of poverty experienced by these mothers may account for elevated depression. Both Blacher et al.'s (1997) study of primarily Mexican American mothers and Magaña's (1999) study of Puerto Rican mothers are based on samples in which the mothers are of extremely low socioeconomic status (SES) and in poor health. Therefore, high rates of depression might be expected in these samples, as much or more as a result of their poverty and health status than due to their special challenges of parenting a child with mental retardation. However, Blacher,
Lopez, Shapiro, and Fusco (1997) later compared their sample of Latina mothers who had a child with mental retardation with a sample of Latina mothers who had only typically developing children. Controlling for health, marital, and SES, they found that having a child with mental retardation was predictive of higher levels of depressive symptoms, suggesting that the caregiving context may be particularly stressful for Latina mothers, above and beyond the effects of poverty (Blacher et al., 1997). One explanation for this finding is that there may be cultural factors affecting the caregiving experience that are contributing to higher levels of depressive symptoms among Latina mothers who provide care to a child with mental retardation.

Two related cultural influences may be expected to contribute to the caregiving experience for Puerto Rican mothers. (Although there are many similarities across Latino groups with regard to cultural values, language, and patterns of assimilation into broader society, there are many differences as well. Because our study is of Puerto Rican mothers, we refer primarily to Puerto Ricans with respect to cultural and environmental factors, recognizing that some of these patterns may be similar to or distinctive from other Latina groups.)

The cultural influences relevant here are familism and gender role differentiation. Familism is a cultural value that refers to nuclear and extended family involvement and interdependence. It involves support from the family, reciprocal obligation to the family, and ties to a collectivist world view in which a family member's individual identity is heavily linked to the group identity (Marin \& Marin, 1991; Sabogal, Marin, Otero, Marin, \& PerezStable, 1987). Related to this cultural value is the role of motherhood in Puerto Rican culture. Within a patriarchal social context, Puerto Rican women are socialized to view motherhood as the most important role for women (Comas-Diaz, 1987).

Gender roles are more differentiated than in non-Latino White cultures, and women are primarily responsible for raising and caring for children (Bassuk et al., 1998; Canino et al., 1987). For Puerto Rican women, the saying la familia primero (family first) links both of these cultural factors (Delgado, 1997). The idea that family comes before all else often is interpreted by Puerto Rican women to mean that family should come before one's own health and emotional needs (Delgado, 1997). The personal well-being of a Puerto Rican woman who is caring for a child with mental retardation is likely 
to suffer in this social context. Further, the overall well-being of the entire Puerto Rican family is likely to impact heavily on the psychological well-being of the Puerto Rican mother, due to her view that the family is a central part of her own identity. We have incorporated these cultural values into the stress and coping model described below.

Adherence to cultural beliefs and values varies based on many factors, including age, migration history, time in country of origin, and level of acculturation (Kalyanpur \& Harry, 1997). Therefore, not all Puerto Rican women would be expected to hold the same beliefs and values. However, as described below, the Puerto Rican mothers in this sample were older, first generation, primarily Spanishspeaking women, and were, therefore, likely to adhere to traditional beliefs and values.

In the present study we extend a previous analysis in which our research group investigated differences and similarities between the Puerto Rican mothers who participated in the current study and a sample of Cuban American mothers who were also providing care to an adult son or daughter with mental retardation (Magaña, Seltzer, Krauss, Rubert, \& Szapocznik, 2002). In that study, a modification of Pearlin, Mullan, Semple, and Skaff's (1990) stress process model was tested, in which family conflict was conceptualized as a secondary role strain that affected caregiver well-being. Building on that model, we hypothesized that if the mother perceives that her family is functioning poorly, she will have higher levels of depressive symptoms than if she perceives that her family is well-functioning. Indeed, we found that having more family problems was a significant predictor of poorer maternal wellbeing for both Latino groups. The relationship between family problems and personal well-being is reflective of the collectivist component of familism: Individual identity and well-being is heavily linked to the group identity and well-being. In the current study, we expand on these empirical findings by investigating whether having greater family problems is also a predictor of depressive symptoms for nonLatina White mothers and, if so, whether it is as strong a predictor as it is for Latina mothers.

Pearlin et al. (1990) specified four domains in the stress process model: the background and context of stress, the sources of stress, the mediators of stress, and the outcomes or manifestations of stress. Sources of stress were divided up into primary stressors and secondary strains in this model. Primary stressors were conceptualized as characteristics of the person with the disability that are known to cause stress; secondary strains were conceptualized as roles and functions that are directly affected by the primary stressors, which, in turn, affect the caregiver's well-being (Pearlin et al., 1990). Based on prior research, we conceptualized that maladaptive behaviors exhibited by the son or daughter with mental retardation would be a primary stressor for their mothers (Greenberg, Seltzer, \& Greenley, 1993; Heller \& Factor, 1991; Seltzer, Greenberg, \& Krauss, 1995). These studies show that maladaptive behaviors can be disruptive to the daily life of mothers (Greenberg et al., 1993; Heller \& Factor, 1991; Seltzer et al., 1995), fathers, and siblings (Essex, 2002; Greenberg, Seltzer, Orsmond, \& Krauss, 1999). We conceptualized family problems as a secondary role strain in order to investigate further how maladaptive behaviors may affect families. As shown in Figure 1, we hypothesized that the primary stressor, maladaptive behaviors, would have a direct effect on the secondary role strain, family problems, which in turn would affect the well-being of the mother. However, we expected that elevated levels of family problems would affect Puerto Rican mothers more than non-Latina White mothers because of the social-cultural context defined by familism and gender role differentiation, as described earlier.

Within the context of this model, we were also interested in examining (a) to what extent sociodemographic characteristics explain differences in maternal depressive symptoms between the two groups and (b) how sociodemographic characteristics may interact with ethnicity in predicting poorer outcomes for Puerto Rican mothers. Previous research on the samples used in the current study has established that the Puerto Rican mothers were less educated, less likely to be married, in poorer health,

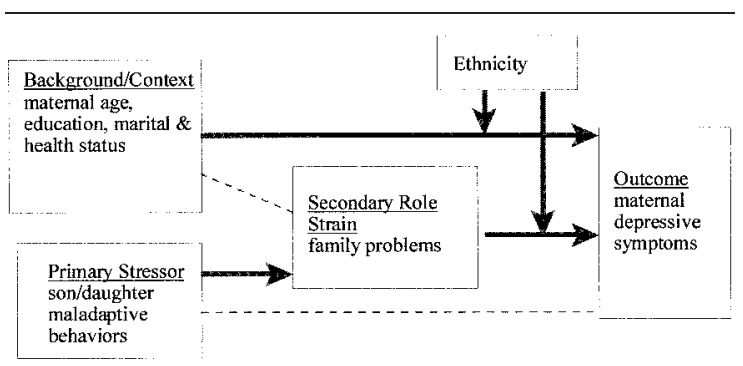

Figure 1 Conceptual framework. Solid lines indicate hypotheses. Dotted lines $=$ variables that are also expected to be related. 
and had higher levels of depressive symptoms than did the non-Latina White mothers (Magaña, 1999; Magaña, Seltzer, \& Krauss, 2002; Seltzer \& Krauss, 1989).

We designed our research questions to test first the overall model for the entire sample of mothers of adults with mental retardation (both Puerto $\mathrm{Ri}$ can and non-Latina White) and then to examine differences between groups within the model. The research questions were: (a) Do family problems mediate the effect of maladaptive behaviors on depressive symptoms (i.e., serve as a secondary role strain) for mothers caring for an adult with mental retardation? (b) To what extent do sociodemographic characteristics explain differences in depressive symptoms between the two groups of mothers? (c) To what extent are the predictors of depressive symptoms different for the two groups of mothers?

\section{Method}

\section{Sample}

The Puerto Rican sample included in this analysis consisted of 66 families who lived in Massachusetts. Participants met three criteria: the mother was the main caregiver for a son or daughter age 18 or older who had mental retardation, the son or daughter lived at home, and the mother and/or the son or daughter were of Puerto Rican descent. All of the mothers were first-generation immigrants (95.5\% were born in Puerto Rico), and all of the adults with mental retardation were of Puerto Rican descent. Two mothers were born in the Dominican Republic but lived in Puerto Rico for a period of time and had a spouse who was Puerto Rican. One mother was born in Columbia and had a spouse from Puerto Rico. On average, the mothers had lived in the United States for 22.1 years at the time of the study. Both Spanish-speaking and Englishspeaking families were eligible to participate in the study; all except one chose to have the interview conducted in Spanish. We note that within-group analyses have been conducted on this sample, and there were no significant relationships between level of acculturation and the key outcome variable (depressive symptoms) in the current study (Magaña, 1999).

Puerto Rican families were recruited with the help of personnel from 14 area offices of the Massachusetts Department of Mental Retardation and community organizations that serve Latino families who have a family member with an intellectual disability. Service providers presented information written in Spanish or English to the families and reviewed it with them. In addition, 8 families were referred by participating sample members. The response rate by those families who were approached about the study was $97 \%$.

The non-Latina White sample included in this analysis consisted of 161 mothers who lived in Massachusetts. These mothers constitute a subsample of mothers from a longitudinal study of 461 families in Wisconsin and Massachusetts who met two criteria at the beginning of the study in 1988: The mother was age 55 or older and the primary caregiver of a son or daughter with mental retardation living at home (see Seltzer \& Krauss, 1989, 1994, for study methodology). The primary method of recruitment was via the formal service system (e.g., the Department of Mental Retardation, the Arc), although snowball sampling was also used. Data were collected 8 times (every 18 months). The sample for the present analysis contained mothers of adult children with -mental retardation who lived at home in Massachusetts at the time of the second point (Time 2) of data collection. Only those mothers who lived in Massachusetts were selected because their social, economic, and service context would be more comparable to the Massachusetts Puerto Rican sample. Time 2 was selected because similar variables were measured in both studies at that time point. We included in this analysis 161 of the original 226 Massachusetts mothers. Of the others, 10 persons with mental retardation had moved out of the home prior to the Time 2 interviews, 29 were not included due to inability to locate or mother refusal, 5 were not included due to the death of the son or daughter with mental retardation, and 21 did not have complete data on the key variables in the model. All of the persons with mental retardation were at least 18 years of age, which was consistent with the Puerto Rican sample.

\section{Measures}

Maternal characteristics included mother's age, marital status, years of education, and health status. Marital status was coded 1 for married and 0 for widowed, divorced, separated, or single. Education level included seven categories from less than high school (0) to completed graduate school (6). Mothers' health status was measured by a question included in the Older Americans' Resources and Ser- 
vices Multidimensional Functional Assessment, in which the mother is asked to rate her own health from excellent (4) to poor (1). The criterion-related validity of this item with a physical examination was reported to be .70 (Fillenbaum, 1978). For ethnicity, a dummy variable was created in which the Puerto Rican cases were coded as 1 and the nonLatina White cases were coded as 0.

Characteristics of the son or daughter with mental retardation included age, gender, level of mental retardation, and number of maladaptive behaviors. For level of mental retardation, mothers were asked whether they had been told their son or daughter was diagnosed with profound (coded 0 ), severe (1), moderate (2), or mild (3) mental retardation. Number of maladaptive behaviors was measured by a scale from the Inventory for Clients in Agency Planning-ICAP (Bruininks, Hill, Weatherman, \& Woodcock, 1986). There were eight items for maladaptive behavior. The mother was asked whether her son or daughter had exhibited the behavior during the past month: hurtful to him/ herself, destructive or hurtful to others, destructive to property, disruptive behavior, unusual or repetitive habits, socially offensive behavior, withdrawn or inattentive behavior, and uncooperative behavior. In order to ensure the validity of the maternal report, specific examples of the maladaptive behavior were solicited from the mother. The ICAP score was the number of maladaptive behaviors reported by the mother.

Family problems was measured by a subscale of the revised version of the Questionnaire on Resources and Stress-F (Friedrich, Greenberg, \& Crnic, 1983). This subscale consists of 20 items answered yes (score of 1 ) or no (score of 0 ), reflecting family well-being and functioning. Sample items are "Other members of the family have to do without things because of __ (son or daughter)." "The constant demands for care for ___ (son or daughter) limit growth and development of someone else in our family." The scale's alpha reliability was .81 for the Puerto Rican sample and .83 for the nonLatino White sample.

Depressive symptoms were measured by the Center for Epidemiologic Studies-Depression Scale (Radloff, 1977). This is a measure of the frequency of 20 depressive symptoms that had occurred over the last week, each rated on a 4-point scale. This scale has been shown to be valid and reliable with many populations and is often used in cross-cultural research (Blacher et al., 1997). The scale's alpha reliability was .89 for the Puerto Rican sample and .83 for the non-Latino White sample.

\section{Data-Collection Procedures}

For both samples, mothers participated in a structured interview in their homes. The non-Latina White mothers also completed self-administered standardized assessments. For the Puerto Rican sample, interviews were conducted by bilingual/ bicultural interviewers, and all items were read to the mothers (because some mothers were not able to read or felt less comfortable with a reading and writing component). Measures were translated using the back-translation method (Kurtines \& Szapocznik, 1995). For the current analyses, the only two measures that were measured differently for the two groups (i.e., collected through a self-administered questionnaire for the non-Latina White sample and via interviews for the Latina sample) were the Center for Epidemiologic Studies-Depression and family problems scales. All other measures used in the current analyses were collected through face-to-face interviews for both groups. Although different methods of administration may limit the comparability of these two measures, one would expect that due to response bias, mothers who were reporting verbally might minimize the extent of problems or symptoms (which would tend to make the two groups more similar). Descriptive data presented in Table 1 suggest that this was not the case: The Puerto Rican mothers reported a higher number of symptoms as compared to the non-Latina White mothers.

\section{Analysis}

Chi-square or $t$ tests were used to determine differences between groups in categorical and continuous demographic characteristics, respectively. Statistical techniques outlined by Baron and Kenny (1986) were used to test mediating and interaction effects within hierarchical regression models. An alpha level of .05 was used for all statistical tests.

Gender of the son or daughter and level of mental retardation were included in preliminary analysis but did not change the pattern of findings. Five members of the Puerto Rican sample did not have data on level of retardation. Therefore, we did not include gender or level of retardation in the final models in order to maintain the maximum statistical power and sample size. 
Table 1 Sample Characteristics

\begin{tabular}{|c|c|c|c|c|c|}
\hline \multirow[b]{2}{*}{ Characteristic } & \multicolumn{2}{|c|}{$\begin{array}{l}\text { Puerto Rican } \\
(n=66)\end{array}$} & \multicolumn{2}{|c|}{$\begin{array}{l}\text { Non-Latino White } \\
\quad(n=161) \\
\end{array}$} & \multirow[b]{2}{*}{ Test } \\
\hline & Mean/\% & $S D$ & Mean/\% & $S D$ & \\
\hline \multicolumn{6}{|l|}{ Mother } \\
\hline Mean age & 57.4 & 8.8 & 68.0 & 6.5 & $t=8.8^{* * *}$ \\
\hline Married $(\%)$ & 39.4 & & 52.8 & & $\chi^{2}=3.4$ \\
\hline High school education (\%) & 21.2 & & 80.7 & & $\chi^{2}=71.5^{* * *}$ \\
\hline Excellent or good health (\%) & 24.2 & & 72.7 & & $\chi^{2}=45.3^{* * *}$ \\
\hline Mean number of family problems & 6.5 & 4.1 & 4.4 & 4.0 & $t=-3.7^{* * *}$ \\
\hline Mean depressive symptoms score & 17.9 & 12.3 & 9.8 & 7.8 & $t=5.0^{* * *}$ \\
\hline \multicolumn{6}{|l|}{ Son/daughter } \\
\hline Male $(\%)$ & 45.5 & & 53.4 & & $\chi^{2}=1.2$ \\
\hline Mean age & 30.1 & 8.2 & 36.0 & 7.0 & $t=5.5^{* * *}$ \\
\hline Severe or profound MR (\%) & $50.8^{\mathrm{a}}$ & & 16.8 & & $\chi^{2}=26.6^{* * *}$ \\
\hline Mean number of maladaptive behaviors & 2.8 & 2.1 & 1.8 & 1.8 & $t=-3.4^{* *}$ \\
\hline
\end{tabular}

${ }^{\mathrm{a}} N=61$.

${ }^{* *} p<.01 .{ }^{* *} p<.001$.

\section{Results}

Table 1 shows a comparison of the two groups on demographic characteristics and study variables. There were striking differences between the two samples on almost every variable. Non-Latina White mothers were older, had higher educational levels, and were in better health than the Puerto Rican mothers. Puerto Rican mothers reported having more family problems and higher levels of depressive symptoms than did non-Latina White mothers. Non-Latino White adults with mental retardation were significantly older, had less severe mental retardation, and exhibited fewer maladaptive behaviors than did the Puerto Rican adults with mental retardation.

Following the analytical method outlined by Baron and Kenny (1986) to address our first research question ("Do family problems mediate the effect of maladaptive behaviors on depressive symptoms for mothers caring for an adult with mental retardation?"), we established that maladaptive behaviors were related to family problems, $r=.40, p$ $<.001$, and that family problems were related to maternal depressive symptoms, $r=.46, p<.001$. We then conducted hierarchical multiple regression analyses to test for mediation effects (see Table 2). At Step 1, having a child with more behavior problems was a significant predictor of maternal depres- sive symptoms (controlling for ethnicity). When the family problems variable was added to the model in Step 2, maladaptive behaviors were no longer a significant predictor of maternal depressive symptoms, but family problems remained significant. Thus, family problems mediated the effect of the child's maladaptive behaviors on maternal depressive symptoms.

In order to test our second research question ("To what extent do sociodemographic characteristics explain differences in depressive symptoms between Puerto Rican and non-Latina White mothers of adults with mental retardation?"), we returned to our hierarchical regression model shown in Table 2. The difference between the two groups on depressive symptoms reported earlier (Puerto Rican mothers had higher levels) was confirmed in Step 1. In Step 3, the maternal characteristics of age, marital status, educational level, and health were added to the model. With these sociodemographic characteristics included, ethnicity was no longer a significant predictor of depressive symptoms. Poor maternal health was the only significant sociodemographic predictor of higher depressive symptoms, along with the family problems variable.

In the analysis to answer the next question ("To what extent are the predictors of depressive symptoms different for the two groups of mothers?"), we focused on the two factors that are sig- 
Table 2 Hierarchical Regression of Depressive Symptoms

\begin{tabular}{lccccc}
\hline Independent variable & Step 1 & Step 2 & Step 3 & Step 4 & Step 5 \\
\hline Ethnicity & $.34^{* * *}$ & $.28^{* * *}$ & .09 & .06 & .00 \\
No. of maladaptive behavior problems & $.14^{*}$ & -.04 & -.02 & -.03 & -.00 \\
Family problems & & $.45^{* * *}$ & $.36^{* *}$ & $.27^{* * *}$ & $.33^{* * *}$ \\
Maternal age & & & -.04 & -.05 & -.04 \\
Maternal marital status & & & -.02 & -.03 & -.03 \\
Maternal education & & -.10 & -.11 & -.10 \\
Maternal health status & & $-.31^{* * *}$ & $-.29^{* * *}$ & $-.15^{*}$ \\
Ethnicity $\times$ Family Problems & & & $.18^{* *}$ & - \\
Ethnicity $\times$ Maternal Health Status & & & & & $.31^{* * *}$ \\
Adjusted $R^{2}$ & $.15^{* * *}$ & $.31^{* * *}$ & $.39^{* * *}$ & $.41^{* * *}$ & $.43^{* * *}$ \\
\hline
\end{tabular}

Note. Pooled sample: Puerto Rican and non-Latina White mothers; $N=167$.

${ }^{*} p<.05 .{ }^{* *} p<.01 .{ }^{* * *} p<.001$.

nificantly predictive of depressive symptoms: family problems and poor maternal health. In order to address this question, we used the method outlined by Baron and Kenny (1986) to test for interaction effects, first between ethnicity and family problems in predicting depressive symptoms and second between ethnicity and maternal health in predicting depressive symptoms. For the investigation of how the effect of family problems on maternal depressive symptoms may differ for the two groups, we constructed an interaction term by taking the product of family problems and ethnicity and added this new variable to the regression model (see Table 2, Step 4). Family problems was centered (the mean of family problems was subtracted from the value in each case) to minimize the correlation with other variables. As shown in Step 4, the interaction term (Ethnicity $\times$ Family Problems) was predictive of depressive symptoms, which supports our hypothesis that family problems has a different effect on depressive symptoms for Puerto Rican mothers and non-Latino White mothers.

Figure 2 provides a graphic depiction of the relationship between family problems and depressive symptoms for each group, controlling for all other variables. As this figure shows, non-Latina White mothers (represented by the solid line) with high family problems had higher depressive symptoms than did those with low family problems, but this difference was much more pronounced in Puerto Rican mothers (represented by the dashed line).

We also created an interaction term between ethnicity and maternal health to determine whether this variable predicts depressive symptoms differ- ently for the two groups of mothers. We found a significant interaction between ethnicity and maternal health status (see Table 2, Step 5). Figure 3 shows that both Puerto Rican and non-Latina White mothers who were in good health had low rates of depression. However, Puerto Rican mothers who were in poor health had extremely elevated levels of depressive symptoms. In contrast, the levels of depressive symptoms for non-Latina White mothers who were in poor health were not substantially higher than for those in good health.

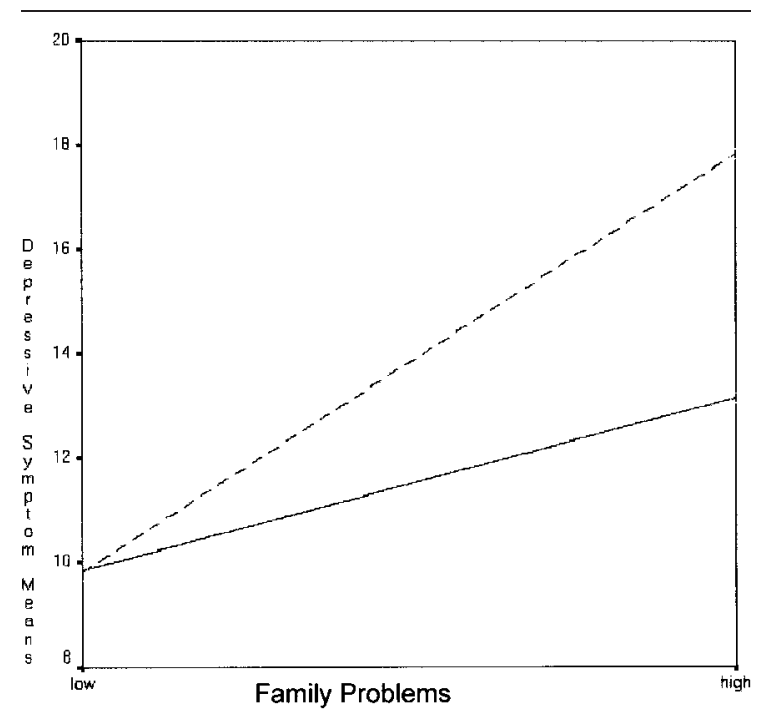

Figure 2 Interaction between ethnicity and family problems. Straight lines $=$ Non-Latina White mothers, dotted lines $=$ Puerto Rican mothers. 


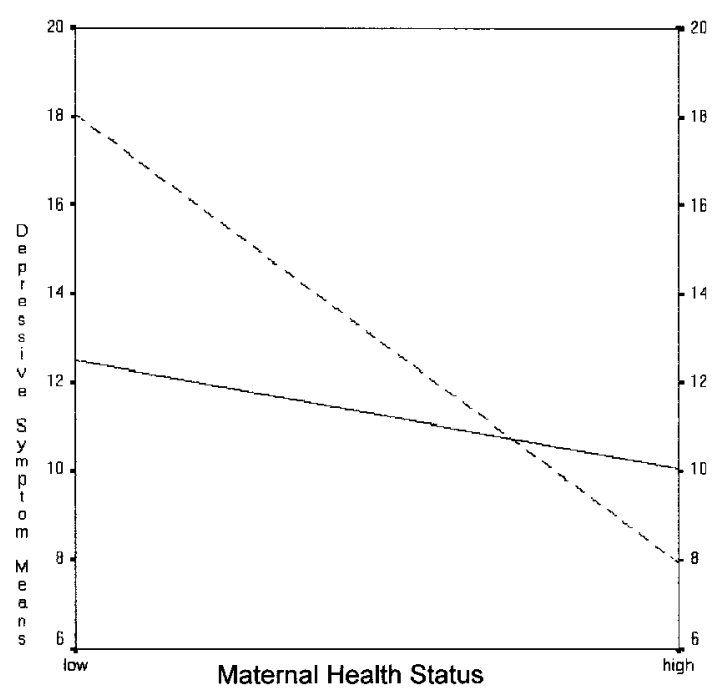

Figure 3 Interaction between maternal health and ethnicity. Straight lines = Non-Latina White mothers; dotted lines = Puerto Rican mothers.

\section{Discussion}

In this study we sought to determine similarities and differences in predictors of depressive symptoms between Puerto Rican and non-Latina White mothers of adults with mental retardation who lived at home. We were interested in reporting on differences between the two groups of mothers, exploring factors that account for differences in depressive symptoms between the two groups and examining whether there are cultural factors (i.e., familism) that interact with caregiving challenges.

Our findings indicate that there are striking differences between the two groups of mothers with respect to their sociodemographic characteristics, characteristics of their son or daughter, and levels of family problems. Non-Latina White mothers were about 10 years older, on average, than the Latina mothers. This difference is due, in part, to differing sample selection criteria (55 and older for non-Latina Whites, whereas there was no maternal age criterion for the Puerto Rican sample). Although older age is generally associated with poorer health and lower income, in this study the younger Puerto Rican mothers were more disadvantaged than were the older non-Latina White mothers. Puerto Rican adults with mental retardation had more behavior problems and more severe levels of retardation than did the children of non-Latina White mothers. Because the majority of families were recruited through the service system, it appears that for Puerto Rican families, those served had children with more severe levels of retardation. Although reasons for this difference are beyond the scope of this study, they may reflect barriers to service access for Latinos, especially those with less severe impairments. Puerto Rican mothers reported comparatively more family problems, which may be attributed to the high number of behavior problems, and more environmental challenges in general (e.g., poverty, low educational level, language problems) as much as culture. As expected from previous findings (Magaña, 1999; Seltzer \& Krauss, 1989), Puerto Rican mothers reported significantly more depressive symptoms. The cumulative effect of these differences suggests substantial disparities in the challenges facing Latina and non-Latina mothers of adults with mental retardation.

Using the stress process model, we found that for both ethnic groups, family problems mediate the effect of maladaptive behaviors of the son or daughter with mental retardation and maternal depressive symptoms. Our findings also suggest that the striking differences in depressive symptoms between the two groups can be accounted for, at least in part, by poor health of the Puerto Rican mothers.

Because Puerto Rican mothers caring for an adult with mental retardation had low SES and because of their focus on caregiving and family wellbeing, they may have been inattentive to their own health needs. In addition to being in poor health, we found that Puerto Rican mothers were more vulnerable emotionally to poor health status: The association between health and depression was more pronounced for the Puerto Rican mothers than it was for the non-Latina White mothers. This finding builds upon our previous analysis in which we compared Puerto Rican mothers to Cuban American mothers (Magaña, Seltzer, Krauss, Rubert, \& Szapocznik, 2002) and, similarly, found that the Puerto Rican mothers were more vulnerable emotionally to health problems than were Cuban American mothers. This difference in the relationship of health status and depression between Puerto Rican and other Latino groups has been documented in other studies (Angel \& Guarnaccia, 1989; Guarnaccia, Good, \& Kleinman, 1990), but is not fully understood.

Our current study also indicates that poor health may not tell the entire story of differences in rates of depression. An important additional factor that appears to contribute to the well-being of Puerto Rican mothers is the well-being of the fam- 
ily. Not only did Puerto Rican mothers report more family problems than did non-Latina White mothers, but the relationship between their family problems and maternal depressive symptoms was stronger. Puerto Rican mothers appear to be more emotionally vulnerable to their perception of how the family is affected by caregiving. Although we were not able to compare cultural values in the two groups, because this was not measured for the nonLatino White sample, we conceptualized the relationship between family problems and depressive symptoms as a partial indicator of the cultural value of familism. This finding suggests that the personal well-being of Puerto Rican mothers may be more influenced by family functioning than that of nonLatina White mothers, thus exhibiting patterns consistent with the collectivist component of familism.

Latinas in the United States have been identified to be at risk for depressive disorders and symptomatology, and Puerto Rican women in the United States appear to be at higher risk than are other groups of Latinas (Cho et al., 1993; Falcon \& Tucker, 2000; Potter et al., 1995; Vasquez, 1994). Results of the present study shed light on how depression may be manifested within a specific context for Puerto Rican women, that of caring for an adult with mental retardation. Within the caregiving context, this study illustrates how cultural and environmental factors (familism and poverty) can contribute to depression in Puerto Rican women and, further, how cultural factors might be taken into account to counteract depression in Puerto Rican women (i.e., promoting personal health care and positive family well-being).

It is important to note the limitations of this analysis. Because of the small size of the Puerto Rican sample, some relationships between variables may not be detected and because members of both samples were volunteers, findings cannot be generalized to the larger population. Also, due to the cross sectional design, the direction of relationships cannot be determined but can only be suggested based on our theoretical model. Another limitation is that there are significant differences between groups with respect to SES and health status of the mothers, maternal age, and differences in number of maladaptive behaviors and severity of disability of the son or daughter. Although we controlled for most of these differences in our analyses, we recognize that the social and economic contexts of the two groups of women are distinctly different, mak- ing comparisons problematic in understanding their divergent experiences.

This study, nevertheless, has several implications for providing supports and services to Puerto Rican caregivers. First, all of the members of the Puerto Rican family need to be considered when providing services to individual members with mental retardation and their caregiver mothers. Understanding how having a family member with mental retardation in the household affects each family member is important for all families, but may be particularly important for Puerto Rican families. Our past research has shown that although many Puerto Rican family members help mothers in day to day caregiving tasks, emotional support has been shown to be more strongly related to maternal psychological well-being than has instrumental support (Magaña, 1999). The current findings suggest that the well-being of the family is also important to maintaining maternal well-being.

In addition, mothers need to be empowered to address their own health and well-being within the context of cultural values to which they may adhere. For example, service providers can acknowledge a mother's commitment to caregiving and family while helping her to understand that she can fulfill this commitment more effectively if she is in better psychological and physical health. In turn, service providers need to understand that by helping to strengthen families, they may be indirectly strengthening the mother's well-being. Consequently, culturally competent services for Puerto Rican mothers of adults with mental retardation should go beyond addressing language issues to understanding how poverty and the cultural commitment to family can affect depression in these women. Services for people with developmental disabilities should aim to connect mothers and their families to needed health and mental health services.

Implications for family research are that we need to move beyond focusing only on the mother as caregiver to more fully investigate family functioning and the well-being of each family member. This includes investigating how family members are affected by having a relative with mental retardation, what their needs are, and how family wellbeing impacts the mother. In future research with cultural and ethnic groups, investigation is needed regarding how such environmental and cultural complexities interact to affect the well-being of the family and, ultimately, the functioning of the adult with mental retardation. 


\section{References}

Aranda, M., \& Knight, B. (1997). The influence of ethnicity and culture on the caregiver, stress and coping process: A sociocultural review and analysis. The Gerontologist, 37, 342-354.

Baca Zinn, M., \& Wells, B. (2000). Diversity within Latino families: New lessons for family social science. In D. H. Demo, K. R. Allen, \& M. A. Fine (Eds.), Handbook of family diversity (pp. 252-273). London: Oxford Press.

Baron, R. M., \& Kinney, D. A. (1986). The moderator-mediator variable distinction in social psychological research: Conceptual, strategic, and statistical considerations. Journal of Personality and Social Psychology, 51, 1173-1182.

Bassuk, E. L., Perloff, J. N., \& Garcia Coll, C. (1998). The plight of extremely poor Puerto Rican and non-Hispanic White single mothers. Social Psychiatry Psychiatric Epidemiology, 33, 326-336.

Blacher, J., Lopez, S., Shapiro, J., \& Fusco, J. (1997). Contributions to depression in Latina mothers with and without children with retardation: Implications for caregiving. Family Relations, 46, 325-334.

Blacher, J., Shapiro, J., Lopez, S., Diaz, L., \& Fusco, J. (1997). Depression in Latina mothers of children with mental retardation: A neglected concern. American Journal on Mental Retardation, 101, 483-496.

Bruininks, R. H., Hill, B. K., Weatherman, R. F., \& Woodcock, R. W. (1986). Inventory for Client and Agency Planning (ICAP). Allen, TX: DLM Teaching Resources.

Canino, G. J., Bird, H. R., Rubio-Stipec, M., \& Bravo, M., (2000). The epidemiology of mental disorders in the adult population of Puerto Rico. Revista Interamericana de Psicologia, 34, 29-46.

Canino, G. J., Bird, H. R., Shrout, P. E., RubioStipec, M., Bravo, M., Martinez, R., Sesman, M., \& Guevara, L. (1987). The prevalence of specific psychiatric disorders in Puerto Rico. Archives of General Psychiatry, 44, 727-735.

Cho, M. J., Móscicki, E. K., Narrow, W. E., Rae, D. S., Locke, B. Z., Regier, D. A. (1993). Concordance between two measures of depression in the Hispanic Health and Nutrition Survey. Social Psychiatry Psychiatric Epidemiology, 28, 156-163.

Comas-Diaz, L. (1987). Feminist therapy with mainland Puerto Rican women. Psychology of Women Quarterly, 11, 461-474.

Delgado, J. (1997). Salud: A Latina's guide to total health—body, mind, and spirit. New York: Harper Collins.

Essex, E. L. (2002). Mothers and fathers of adults with mental retardation: Feelings of intergenerational closeness. Family Relations, 51, 156165.

Falcon, L., \& Tucker, K. (2000). Prevalence and correlates of depressive symptoms among Hispanic elders in Massachusetts. Journals of Gerontology: Social Sciences, 55B, S108-S116.

Fillenbaum, G. (1978). Validity and reliability of the Multidimensional Functional Assessment Questionnaire. In Duke University Center for the Study of Aging and Human Development (Ed.), Multidimensional functional assessment: The OARS methodology. Durham, NC: Duke University, Center for the Study of Aging and Human Development.

Friedrich, W., Greenberg, M., \& Crnic, K. (1983). A short form of the questionnaire on resources and stress. American Journal of Mental Deficiency, 88, 345-351.

Gatz, M., \& Hurwicz, M. (1990). Are older people more depressed? Cross sectional data on Center for Epidemiological Studies Depression Scale factors. Psychology and Aging, 5, 284-290.

Greenberg, J. S., Seltzer, M. M., \& Greenley, J. R. (1993). Aging parents of adults with disabilities: The gratifications and frustrations of laterlife caregiving. The Gerontologist, 33, 542-550.

Greenberg, J. S., Seltzer, M. M., Orsmond, G. I., \& Krauss, M. W. (1999). Siblings of adults with mental illness or mental retardation: Current involvement and expectation of future caregiving. Psychiatric Services, 50, 1214-1219.

Heller, T., \& Factor, A. (1991). Permanency planning for adults with mental retardation living with family caregivers. American Journal on Mental Retardation, 96, 163-176.

Kalyanpur, M., \& Harry, B. (1997). A posture of reciprocity: A practical approach to collaboration between professionals and parents of culturally diverse backgrounds. Journal of Child and Family Studies, 6, 487-509.

Krauss, M. W., \& Seltzer, M. M. (1993). Current well-being and future plans of older caregiving mothers. Irish Journal of Psychology, 14, 48-63.

Kurtines, W. M., \& Szapocznik, J. (1995). Cultural competence in assessing Hispanic youths and 
families: Challenges in the assessment of treatment needs and treatment evaluation for Hispanic drug abusing adolescents. In E. Rahdert \& D. Czechowicz (Eds.), Adolescent drug abuse: Clinical assessment and therapeutic interventions (NIDA Research Monograph No. 156, NIH Publication No. 95-3908, pp. 172-189). Rockville, MD: National Institute on Drug Abuse (NIDA).

Magaña, S. (1999). Puerto Rican families caring for an adult with mental retardation: The role of familism. American Journal on Mental Retardation, 104, 466-482.

Magaña, S., Seltzer, M., \& Krauss, W. (2002). Service utilization patterns of adults with intellectual disabilities: A comparison of Puerto Rican and non-Latino White families. Journal of Gerontological Social Work, 37 (3/4).

Magaña, S., Seltzer, M., Krauss, M., Rubert, M., \& Szapocznik, J. (2002). Well-being and family role strain among Cuban American and Puerto Rican mothers of adults with mental retardation. Journal of Human Behavior in the Social Environment, 5, 31-55.

Marin, G., \& Marin, B. (1991). Research with Hispanic populations. Newbury Park: Sage.

Pearlin, L., Mullan, J., Semple, S., \& Skaff, M. (1990). Caregiving and the stress process: An overview of concepts and their measures. The Gerontologist, 30, 583-594.

Potter, L. B., Rogler, L. H., \& Moscicki, E. K. (1995). Depression among Puerto Ricans in New York City: Hispanic Health and Nutrition Examination Survey. Social Psychiatry Psychiatric Epidemiology, 30, 185-193.

Radloff, L. (1977). The CES-D scale: A self-report depression scale for research in the general population. Applied Psychological Measurement, 1, 385-401.

Sabogal, F., Marin, G., Otero-Sabogal, R., Marin, B. V., \& Perez-Stable, E. J. (1987). Hispanic familism and acculturation: What changes and what doesn't. Hispanic Journal of Behavioral Science, 9, 397-412.

Seltzer, M. M., Greenberg, J. S., \& Krauss, M. W. (1995). A comparison of coping strategies of aging mothers of adults with mental illness and mental retardation. Psychology and Aging, 10, 64-75.

Seltzer, M. S., \& Krauss, M. W. (1989). Aging par- ents with adult mentally retarded children: Family risk factors and social support. American Journal on Mental Retardation, 94, 303-312.

Seltzer, M. M., \& Krauss, M. W. (1994). Aging parents with coresident adult children: The impact of lifelong caregiving. In M. M. Seltzer, M. W. Krauss, \& M. P. Janicki (Eds.), Life course perspectives on adulthood and old age (pp. 3-19). Washington, DC: American Association on Mental Retardation.

Shrout, P. E., Canino, G. J., Bird, H. R., RibioStipec, M., Bravo, M., \& Burnam, M. A. (1992). Mental health status among Puerto Ricans, Mexican Americans, and non-Hispanic Whites. American Journal of Community Psychology, 20, 729-752.

Vasquez, M. (1994). Latinas. In L. Comas-Díaz \& B. Greene (Eds.), Women of color: Integrating ethnic and gender identities in psychotherapy (pp. 114-138). New York: Guilford Press.

Received 4/26/02, first decision 10/14/02, accepted 12/09/02.

Editor-in-charge: Steven J. Taylor

Support for the preparation of this manuscript was provided by a grant from the National Institute on Aging (R01 AG08728), a minority supplement grant from the National Institute on Mental Health (RO1 MH55928), and grants from the National Institute of Child Health and Human Development (T32 HD 07194, T32 HD07489), and the Merck Scholars II Program. Support was also provided by the Starr Center on Mental Retardation, Heller School at Brandeis University, and the Waisman Center at the University of Wisconsin-Madison. We also give special acknowledgment to the Massachusetts families who participated in the study.

\footnotetext{
Authors:

Sandra Magaña, PhD (E-mail: MAGANA@ Waisman.Wisc.Edu), Assistant Professor, School of Social Work and Waisman Center; and Marsha Mailick Seltzer, PhD, Professor, School of Social Work, and Director, Waisman Center, University of Wisconsin-Madison, 1500 Highland Ave., Madison, WI 53705-2280. Marty Wyngaarden Krauss, PhD, John Stein Professor of Disability Research, Heller School, Brandeis University, PO Box 9110, Waltham, MA 02254-9110.
} 